\section{Multistate Outbreak of an Emerging Burkholderia cepacia Complex Strain Associated With Contaminated Oral Liquid Docusate Sodium}

To the Editor-We read with interest the article by Marquez et $\mathrm{al}^{1}$ in which the authors report an epidemiologic investigation into a Burkholderia cepacia complex outbreak among pediatric patients. This prompted a national recall of oral liquid docusate $^{\mathrm{a}}$ in 2016 . $^{2}$ Here, we describe a similar outbreak in our pediatric intensive care unit (PICU) from May 19, 2017 to July 30, 2017, that prompted another national product recall.,

In May 2017, we were notified by our clinical microbiology laboratory of 3 patients in the PICU, all infants with multiple chronic medical conditions who had cultures ( 1 from blood and 2 from tracheal aspirates) positive for B. cepacia within the same week. A thorough review of the patients' history, exposures and medications was conducted. Given the commonality of mechanical ventilation, we focused on possible respiratory sources of infection or exposure to previously recalled oral liquid docusate products. ${ }^{2}$ We did not identify common respiratory medications or equipment exposures among cases and all environmental cultures were negative for B. cepacia.

As part of the outbreak investigation, bacterial isolates from cases were analyzed by the Maryland Department of Health $(\mathrm{MDH})$ using pulsed-field gel electrophoresis and by the Burkholderia cepacia Research Laboratory and Repository (BCRLR) at the University of Michigan using repetitive extragenic palindromic polymerase chain reaction (rep-PCR). The isolates from the 3 cases were closely related, suggesting a common exposure. The BCRLR designated this strain "1072," which was different from the B. cepacia strain associated with the 2016 docusate outbreak. ${ }^{1}$ Additionally, external communications revealed that 2 critically ill infants at another hospital were infected with a similar strain of B. cepacia, raising concern for a multistate outbreak.

Five weeks after the initial cases, another critically ill infant with a prolonged PICU admission had a respiratory culture that grew B. cepacia strain 1072. Investigators from $\mathrm{MDH}$ visited our facility to review infection control practices and helped coordinate investigations among our hospital, the Centers for Disease Control and Prevention (CDC), and other hospitals with suspect Burkholderia cases. Lists of medications dispensed to all cases were compared among the affected hospitals. Two months after the initial cases, a fifth case was identified in a ventilated PICU patient whose urine culture grew B. cepacia strain 1072. Due to concerns for ongoing local transmission, universal contact precautions for all PICU patients was initiated. Our institution debuted real-time video surveillance of healthcare workers behavior with instant verbal feedback on hand hygiene compliance and adherence to standard and contact precautions. We also conducted targeted surveillance cultures. No additional respiratory cultures were positive; however, 2 stool cultures were positive for B. cepacia strain 1072. The investigation timeline is depicted in Figure 1.

A CDC epidemiologist noted that the National Drug Code (NDC) for oral liquid docusate from the manufacturer associated with the 2016 docusate outbreak (NDC 0536-0590-85) appeared in medication administration data for case patients at another hospital, and an infection preventionist at our hospital determined that docusate with this NDC had also been administered to case patients at our hospital. ${ }^{1}$ Based on this finding, we provided opened and unopened bottles of docusate to the CDC and US Food and Drug Administration for culture. In late July, we were informed that an open bottle of liquid docusate grew B. cepacia, after which all docusate products from the implicated manufacturer were removed from our patient care areas and pharmacies. Burkholderia cepacia isolated from the opened docusate bottle was confirmed as strain 1072, implicating the contaminated oral liquid docusate as the likely common source of infections at the hospitals with affected patients. In early August, there was a voluntary recall of liquid docusate due to contamination concerns ${ }^{3}$ and later that week, a broader recall of additional liquid medications took place. ${ }^{4,5}$

Respiratory and bloodstream infections after exposure to an enteral medication are somewhat unexpected. We speculate that B. cepacia might have colonized and infected infants via aspiration of enteral fluids, translocation across the enteric mucosa or migration into the genitourinary tract from the gastrointestinal tract, putting the most critically ill patients at highest risk. ${ }^{1}$

We learned several unique lessons from this experience: (1) Identification of respiratory sites of infection should not limit investigation to only respiratory-related sources. (2) Despite early identification of a bacterial strain different from that implicated in previously recalled products, outbreak investigators should maintain a high level of suspicion and use NDC codes to identify common products or manufacturers. (3) Rapid reporting to and involvement of federal, state and local agencies in outbreak investigations can help identify a common exposure and support identification of pathogenic bacteria in medications and other products. In addition, access to a national reference laboratory for molecular typing was invaluable in this investigation and outbreak confirmation. (4) While there are no national guidelines on disclosure to patients and families in outbreak investigations, clinicians should provide clear and effective communication. ${ }^{6}$ Throughout this

\footnotetext{
a Note that docusate refers to oral liquid docusate sodium.
} 

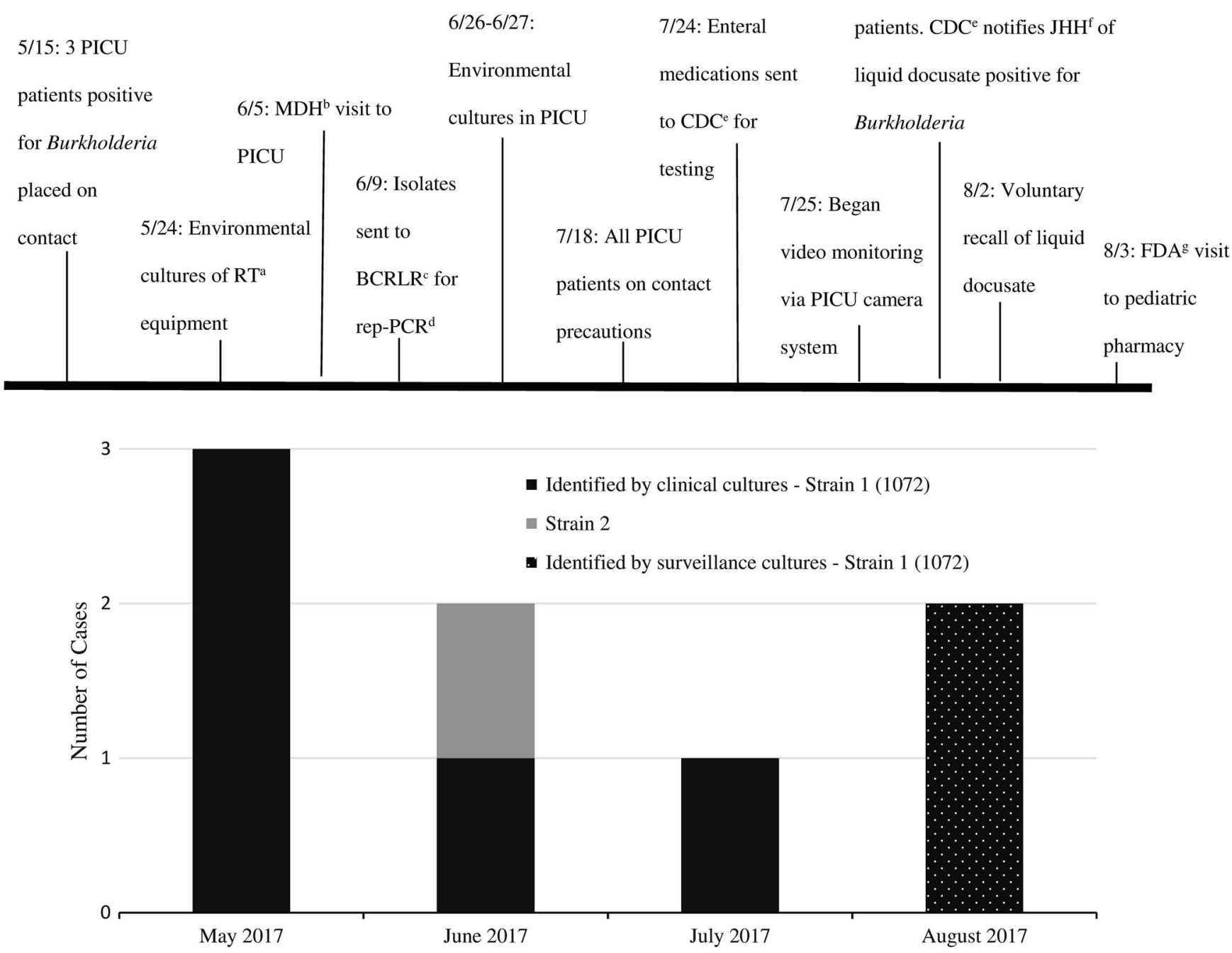

FIGURE 1. Burkholderia cepacia investigation timeline. (a) Respiratory Therapy. (b) Maryland Department of Health. (c) Burkholderia cepacia Research Laboratory and Repository. (d) Repetitive extragenic palindromic PCR. (e) Centers for Disease Control and Prevention. (f) Johns Hopkins Hospital. (g) U.S. Food and Drug Administration.

investigation, we strived to be transparent with patients' families. We personally spoke with the families of the affected patients and provided all families in the PICU with an informational handout, which was well received.

Lastly, while some B. cepacia outbreaks have never been linked to a known source, ${ }^{7}$ B. cepacia has relatively low occurrence in non-cystic fibrosis patients. Therefore, identification of a cluster of infections, particularly those caused by an identical strain, should raise suspicion for possible product contamination or infection control deficiencies, and should prompt further investigation.

\section{ACKNOWLEDGMENTS}

The authors thank Mary-Beth Griffith, LeAnn McNamara, Matt Crist, Janet Glowicz, Pam Dodge, Stacey Mann, Alice Hsu, Mark Romagnoli, Roshni Mathew, the Division of Medical Microbiology, the Department of Pharmacy, the
Department of Hospital Epidemiology and Infection Control, Maryland Department of Health Laboratories administration, and the entire Johns Hopkins Hospital PICU staff for their dedication to patient safety and helping to quickly contain this outbreak.

Financial support: No financial support was provided relevant to this article. Potential conflicts of interest: All authors report no conflicts of interest relevant to this article.

\author{
Ibukunoluwa C. Akinboyo, MD; ${ }^{1, a}$ \\ Anna C. Sick-Samuels, MD MPH; ; \\ Emily Singeltary, $\mathrm{MPH}{ }^{2}$ \\ James Fackler, $\mathrm{MD} ;^{3}$ \\ Judith Ascenzi, DNP; ${ }^{4}$ \\ Karen C. Carroll, MD; \\ Yvonne Maldonado, $\mathrm{MD}{ }^{6}$ \\ Richard B. Brooks, MD; ${ }^{7,8}$ \\ Isaac Benowitz, MD; ${ }^{8}$
}




\section{Lucy E. Wilson, MD; ${ }^{7}$ \\ John J. LiPuma, MD; ${ }^{9}$ Aaron M. Milstone, MD, MHS ${ }^{1,2}$}

Affiliations: 1. Department of Pediatrics, Division of Pediatric Infectious Diseases, Johns Hopkins University School of Medicine, Baltimore, Maryland; 2. Department of Hospital Epidemiology and Infection Control, The Johns Hopkins Hospital, Baltimore, Maryland; 3. Department of Anesthesia, Division of Pediatric Intensive Care Unit, Johns Hopkins University School of Medicine, Baltimore, Maryland; 4. Department of Pediatric Nursing, The Johns Hopkins Hospital, Baltimore, Maryland; 5. Department of Pathology, Division of Medical Microbiology, Johns Hopkins University School of Medicine, Baltimore, Maryland; 6. Division of Pediatric Infectious Disease, Stanford University School of Medicine, Stanford, California; 7. Infectious Disease Epidemiology and Outbreak Response Bureau, Maryland Department of Health, Baltimore, Maryland; 8. Division of Healthcare Quality Promotion, Centers for Disease Control and Prevention, Atlanta, Georgia; 9. Department of Pediatrics and Communicable Disease, University of Michigan Medical School, Ann Arbor, Michigan.

Address correspondence to Ibukunoluwa Akinboyo, 200 North Wolfe Street, Room 3155, Baltimore, MD 21287 (ibukun@jhmi.edu).

${ }^{a}$ Contributed equally as first authors.

Infect Control Hosp Epidemiol 2018;39:237-239

(c) 2018 by The Society for Healthcare Epidemiology of America. All rights reserved. 0899-823X/2018/3902-0020. DOI: 10.1017/ice.2017.265

\section{REFERENCES}

1. Marquez L, Jones KN, Whaley EM, et al. An outbreak of Burkholderia cepacia complex infections associated with contaminated liquid docusate. Infect Control Hosp Epidemiol 2017;38:567-573.

2. US Food and Drug Administration (FDA). FDA updates on multistate outbreak of Burkholderia cepacia infections. FDA website. https://www.fda.gov/Drugs/DrugSafety/ucm511527. htm. Published 2016. Accessed November 5, 2017.

3. Office of Regulatory Affairs, US Food and Drug Administration (FDA). Safety alerts—rugby laboratories issues voluntary nationwide recall of diocto liquid and diocto syrup manufactured by PharmaTech, LLC, due to possible product contamination. FDA website. https://www.fda.gov/safety/recalls/ucm569967. htm. Published 2017. Accessed November 5, 2017.

4. Office of the Commissioner, US Food and Drug Administration (FDA). Safety alerts for human medical products: diocto liquid and diocto syrup by rugby laboratories: recall— possible product contamination. FDA website. https://www.fda.gov/Safety/MedWatch/ SafetyInformation/SafetyAlertsforHumanMedicalProducts/ucm 570014.htm. Published 2017. Accessed November 5, 2017.

5. Office of Regulatory Affairs, US Food and Drug Administration (FDA). Safety alerts: voluntary nationwide recall of all liquid products manufactured by Pharmatech LLC and distributed by Leader Brand, Major Pharmaceuticals, and Rugby laboratories due to possible product contamination. FDA website. https:// www.fda.gov/Safety/Recalls/ucm571001.htm. Published 2017. Accessed November 5, 2017.

6. Daugherty Biddison L, Berkowitz KA, Courtney B, et al. Care of the critically ill and injured during pandemics and disasters: CHEST consensus statement. Chest 2014;146:e145S-e155S.

7. Siddiqui AH, Mulligan ME, Mahenthiralingam E, et al. An episodic outbreak of genetically related Burkholderia cepacia among non-cystic fibrosis patients at a university hospital. Infect Control Hosp Epidemiol 2001;22:419-422.
A Colonization Outbreak of PenicillinSusceptible mecA-Positive Staphylococcus aureus in a Neonatal Ward of Children's Hospital

To the Editor-We experienced a colonization outbreak of penicillin-susceptible and mecA-positive Staphylococcus aureus strain in neonatal ward. After implementation of strict precautions and decolonization, the outbreak was terminated. To our knowledge, this is the first report of penicillinsusceptible MRSA outbreak in a neonatal ward.

Nagano Children's Hospital is a tertiary pediatric hospital located in a rural area of Japan with 42 beds in the neonatal ward. Active weekly surveillance cultures of nares of inpatients of the neonatal ward have been carried out since the 1990s, especially for monitoring methicillin-resistant Staphylococcus aureus (MRSA). In recent years, the proportion of MRSA carriage in the neonatal ward has been approximately 0 to $5 \%$.

In late July 2016, our surveillance system noticed an unusual surge in the colonization rate of methicillin-susceptible S. aureus (MSSA) in the neonatal ward. Detailed investigations revealed that this $S$. aureus strain has unique characteristics; namely, it is uniformly susceptible to penicillin but hetero-resistant to oxacillin and cefoxitin. Genetic analysis also revealed that this strain possesses the mecA gene; therefore, this strain was reassessed as MRSA, according to Clinical and Laboratory Standards Institute (CLSI) criteria. ${ }^{1}$

The infection control team had emergency meetings and alerted healthcare workers throughout the hospital about the outbreak. The campaign for reinforcement of hand hygiene with contact precautions, strict isolation, and cohorting the patients was carried out. However, by the end of August, the colonization rate reached its the highest level (12 of 43 patients, 28\%). We then decided to implement MRSA decolonization with mupirocin ointment. In total, 17 patients (12 penicillin-susceptible [PS] MRSA patients and 5 'ordinal' MRSA carrier patients) had undergone the decolonization; 10 of 13 patients $(76.9 \%)$ were confirmed as decolonized (defined as negative results for 2 consecutive cultures). Furthermore, 3 patients were not decolonized, and the other 4 patients were discharged before follow-up cultures were performed.

After these interventions, the carriage rate of PS-MRSA decreased, and no new cases of colonization were reported for 2 consecutive weeks. In late October, we declared that the outbreak had ended. Fortunately, there were no serious infections due to this PS-MRSA during this outbreak.

The outbreak strain of PS-MRSA did not yield typical cultures on MRSA-specific chromogenic media (CHROMagar II, Becton-Dickinson, Japan); on this selective medium, it yielded only a few slow-growing colonies, and sometimes the strain did not yield a culture on the medium. Antimicrobial susceptibility test showed resistance to gentamicin, erythromycin, and levofloxacin but susceptibility to vancomycin. The minimum 